\title{
EXPERIENCIA CLINICA Y RESULTADOS EN EL TRATAMIENTO DE 4402 PACIENTES CON CÁLCULOS URINARIOS ÚNICOS CON EL LITOTRITOR EXTRACORPÓREO DORNIER MFL-5000.
}

\author{
Alberto Palacios, Filipe Rodrigues, Mário Jorge Soares, Adérito Morais, Apolinário Mendes y \\ Jorge Pinheiro.
}

Servicio de Urología. Centro Hospitalar Vila Real-Peso da Régua. Vila Real. Portugal.

\begin{abstract}
Resumen.- OBJETIVO: Presentamos la casuística de nuestro servicio en el tratamiento de la litíasis urinaria así como la efectividad del litotritor Dornier MFL-5000

MÉTODOS: Tratamos en 12 años un total de 6602 pacientes con un número total de sesiones de litotricia de 17120. Realizamos el estudio del éxito del tratamiento de los 4402 pacientes que presentaban un cálculo, según el tamaño y la localización.
\end{abstract}

RESULTADOS: No hubo complicaciones mayores y apenas 43 pacientes tuvieron calle litiásica. Realizamos el tratamiento de 4402 cálculos. Para todos los cálculos independientemente de su localización y tamaño supone que $2615(59,4 \%)$ se han tratado en una sesión, $801(18,2 \%)$ en dos, $379(8,6 \%)$ en tres, $213(4,8 \%)$ en cuatro y $372(8,4 \%)$ en cinco o más sesiones.

Alberto Palacios Hernández Rua Mayor, N-43-47, 3-B 37002 Salamanca. (España). aphmmx5@hotmail.com

Trabajo recibido: 12 de diciembre 2006.
CONCLUSIONES: El litotritor MFL-5000 ha permitido el tratamiento de la litiasis urinaria en todas las edades, con unos porcentajes de retratamiento y un número de tratamientos por cálculo similar a otros autores, sin necesidad de analgesia ni medidas anestésicas, salvo en apenas aquellos pacientes de menor edad.

Palabras clave: MFL-5000. Litotricia. Cálculos urinarios.

Summary.- OBIECTIVES: To report the series of our Urology Department in the treatment of urinary stone disease with extracorporeal lithotripsy as well as the effectiveness of the Dornier lithotripter MFL-5000.

METHODS: 6.602 patients were treated by extracorporeal lithotripsy during 12 years with a total of 17.120 sessions. We examined the treatment outcome of 4.402 patients with a solitary stone, accordingly to stone size and localization.

RESULTS: There were no major complications and only 43 patients experienced "calle litiásica". We treated 4.402 stones. For all stones, independently of size and localization, 2615 (59.4\%) were treated in one session, 801 (18.2\%) required two sessions, 379 (8.6\%) 3 sessions, 213 (4.8\%) 4 sessions and 373 (8.4\%) 5 or more sessions.

CONCLUSION: The Dornier MFL-5000 lithotripter successfully treated urinary stone disease in patients of all age groups with a retreatment rate and number of sessions per stone similar to other studies, without analgesia or anesthetic procedures, except in the younger age group.

Keywords: MFL-5000. Urinary stone. Lithotripsy. 


\section{INTRODUCCIÓN}

Con la introducción del litotritor extracorpóreo por ondas de choque en el año 1980, cambió radicalmente el tratamiento de los cálculos urinarios (1). Este avance tecnológico ha remplazado la necesidad de cirugía abierta en la mayoría de los pacientes, reduciendo la morbilidad, estadía hospitalaria y el coste del tratamiento.

Con los años, los litotritores han avanzado intentando mejorar la eficacia y disminuyendo la morbilidad del paciente. Así por un lado, ha habido un avance tecnológico de los generadores electrohidraúlicos, y por otro, el propio avance ha llevado a la introducción de nuevos generadores del tipo piezoeléctrico y electromagnético, eliminando la necesidad de anestesia al reducir la intensidad de la energía aplicada, disminuyendo el coste debido a sus requisitos de mantenimiento y disminuyendo el índice de complicaciones para el paciente y aumentando el índice de éxito de fragmentación. En la mayoría de los litotritores se han añadido a los rayos $X$, un ecógrafo, disponiendo así de máquinas móviles. Se han incorporado mesas multiuso que permiten realizar procedimientos endoscópicos o radiológicos auxiliares para un tratamiento integral de la litíasis (2).

El 11 de octubre del año 1994 el Hospital de Vila Real pone en funcionamento en nuestro Servicio el litotritor MFL-5000, que dispone de un generador electrohidraúlico, de rayos $X$ y ultrasonidos, y de una mesa multiuso.

Después de 12 años utilizando el litotritor extracorpóreo MFL-5000 y con la entrada en nuestro servicio de un nuevo litotritor electomagnético (Dornier - DOLLY-S), decidimos publicar nuestra casuística. En este artículo realizamos un estudio exhaustivo de los pacientes que presentaban un cálculo.

\section{MATERIAL Y MÉTODOS}

En nuestra institución hemos tratado un total de 6.602 pacientes realizando un número total de sesiones de 17.120, con una media de sesiones por paciente de 2,8. Un total de 4.402 pacientes presentaban cálculo único, de esos pacientes 1.843 eran hombres y 2.559 mujeres. Todas las litiasis fueron tratadas con el litotritor de segunda generación MFL-5000. Éste dispone de un generador de ondas de choque electrohidraúlico de $60 \mathrm{nF}$ de capacidad, un rango de presión de 600-1200 bar y una apertura de elipsoide mayor que sus predecesores $\mathrm{HM}-3$ y HM-4 originales, lo que supone una mayor área de penetración cutánea disminuyendo así la sensación dolorosa. Se completa con un doble sistema de localización de la litíasis con radioscopia y ultrasonidos, lo que permite con este último, el tratamiento de litiasis radiotransparentes, y con una mesa multiuso que permite realizar exploraciones endoscópicas y radiológicas. El número de ondas de choque no excedió los 3.000 en cálculos renales ni de los 4.000 en cálculos ureterales o vesicales por sesión. La tensión por onda de choque no sobrepasó los $23 \mathrm{Kv}$ en riñón ni los $28 \mathrm{Kv}$ en ureter o vejiga. No fue necesario utilizar anestesia en la mayoría de los casos. Cuando hubo fracaso en la fragmentación total del cálculo durante la primera sesión de litotricia, una segunda y si fue necesario más sesiones de litotricia se desarrollaron, con un intervalo entre ambas de 3 a 4 semanas, dependiendo de la localización del cálculo.

Las características del cálculo (tamaño, localización) fueron recogidas al inicio de la sesión de litotricia. Evaluamos tres localizaciones posibles, renal, ureteral y vesical. Consideramos cálculos coraliformes aquellos que ocupan parte de la pelvis renal alcanzando uno, dos o varios brazos caliciales. Antes del tratamiento de pacientes con cálculos coraliformes colocamos un cateter ureteral tipo "pig-tail" de 7. 8 French.

TABLA I. TAMAÑO CÁlCULO RENAL (PELVIS RENAL).

\begin{tabular}{|c|c|c|c|c|c|c|c|}
\hline $\mathbf{N}^{\circ}$ SESIONES & $\mathbf{0 - 9} \mathbf{~ m m}$ & $\mathbf{1 0 - 1 9} \mathbf{~ m m}$ & $\mathbf{2 0 - 2 9} \mathbf{~ m m}$ & $\mathbf{3 0 - 3 9} \mathbf{~ m m}$ & $\mathbf{4 0 - 4 9} \mathbf{~ m m}$ & $\mathbf{5 0 - 5 9} \mathbf{~ m m}$ & $\mathbf{6 0 - 6 9} \mathbf{~ m m}$ \\
\hline & $\mathrm{N}=923$ & $\mathrm{~N}=1192$ & $\mathrm{~N}=334$ & $\mathrm{~N}=77$ & $\mathrm{~N}=26$ & $\mathrm{~N}=11$ & $\mathrm{~N}=5$ \\
\hline 1 & $80,5 \%$ & $48,3 \%$ & $29 \%$ & $9 \%$ & $11,5 \%$ & $27,2 \%$ & $0 \%$ \\
\hline 2 & $92,2 \%$ & $75,4 \%$ & $53 \%$ & $9 \%$ & $27 \%$ & $36 \%$ & $40 \%$ \\
\hline 3 & $97,5 \%$ & $87,3 \%$ & $66,4 \%$ & $27,7 \%$ & $34,6 \%$ & $63 \%$ & $40 \%$ \\
\hline
\end{tabular}

Número total de pacientes: 2.568 
TABLA II. TAMAÑO CÁlCULO URETERAL.

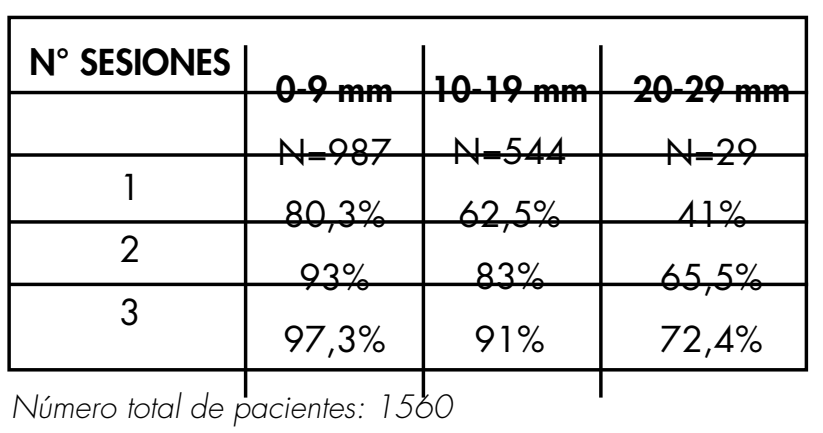

Posterior al tratamiento, pacientes con fragmentos residuales $\leq 4 \mathrm{~mm}$ o libres de fragmentos se consideraron curados. Los pacientes con fragmentos residuales $>4 \mathrm{~mm}$ fueron tratados de nuevo o considerados para otro tratamiento alternativo.

\section{RESULTADOS}

De los 4.402 pacientes monocálculo, 1.843 corresponden al sexo masculino $(41,86 \%)$ y 2.559 al sexo femenino $(58,13 \%)$. Las edades de los pacientes eran comprendidas entre 2 y 95 años, con una edad media de 48,2 años.

La localización del cálculo fue la siguiente: renal $2.568(58,3 \%)$, ureteral $1.560(35,4 \%)$, vesical $59(1,3 \%)$ y coraliformes $215(4,8 \%)$.

Con respecto al tamaño de las litiasis, independientemente de su localización, 1.912 (43,4\%) tenían un diámetro igual o inferior a $1 \mathrm{~cm}$, en 1.748 $(39,7 \%)$ éste estaba entre 1 y $2 \mathrm{~cm}, 378(8,6 \%)$ entre 2 y $3 \mathrm{~cm}, 125(2,8 \%)$ entre 3 y $4 \mathrm{~cm}, 91(2,1 \%)$ entre 4 y $5 \mathrm{~cm}, 63(1,4 \%)$ entre 5 y $6 \mathrm{~cm}, 39(0,8 \%)$ entre
6 y $7 \mathrm{~cm}, 32(0,7 \%)$ entre 7 y $8 \mathrm{~cm}, 12(0,3 \%)$ entre 8 y $9 \mathrm{~cm}, 1(0,02 \%)$ entre 9 y $10 \mathrm{~cm}$ y $1(0,02 \%)$ entre 10 y $11 \mathrm{~cm}$.

Realizamos el tratamiento de 4.402 cálculos. Para todos los cálculos, independientemente de su localización y tamaño, supone que $2.615(59,4 \%)$ se han tratado en una sesión, 801 (18,2\%) en dos, 379 $(8,6 \%)$ en tres, $213(4,8 \%)$ en cuatro y $372(8,4 \%)$ en cinco o más sesiones.

En las Tablas I-IV mostramos el porcentaje de pacientes tratados con éxito en las diferentes sesiones, con respecto al tamaño y a su localización.

Los kilovoltajes (dv) medios utilizados en cada sesión variaron entre 14 y 28 kv con una media de 20,03 kv. La duración media de una sesión de litotricia fue de 75 minutos.

Fue necesario anestesia general en 2 niños con edades de 24 meses y 5 años, realizamos litotricia en otros dos niños de 5 años, sin necesidad de anestesia. En los primeros 900 casos realizábamos analgesia con petidina, al observar que los pacientes no tenían dolor decidimos no incluir ninguna medida de analgesia, el éxito de fragmentación no varió.

\section{DISCUSIÓN}

Nuestra experiencia con el litotritor MFL5000 , durante un periodo de 12 años y con el tratamiento de 6.602 pacientes y un número de sesiones de 17.120, con una media global de 2,8 sesiones por paciente, es uno de las casuísticas mayores que existen en la literatura para este litotritor. Desde la introducción de los litotritores, las técnicas quirúrgicas abiertas para el tratamiento de cálculos urinarios han sido relegadas a un segundo plano en nuestra práctica (2).

TABLA III. TAMAÑO CÁLCULO VESICAL.

\begin{tabular}{|c|c|c|c|c|c|c|c|}
\hline $\mathbf{N}^{\circ}$ SESIONES & $\mathbf{0 - 9} \mathbf{~ m m}$ & $\mathbf{1 0 - 1 9} \mathbf{~ m m}$ & $\mathbf{2 0 - 2 9} \mathbf{~ m m}$ & $\mathbf{3 0 - 3 9} \mathbf{~ m m}$ & $\mathbf{4 0 - 4 9} \mathbf{~ m m}$ & $\mathbf{5 0 - 5 9} \mathbf{~ m m}$ & $\mathbf{6 0 - 6 9} \mathbf{~ m m}$ \\
\hline & $\mathrm{N}=2$ & $\mathrm{~N}=12$ & $\mathrm{~N}=11$ & $\mathrm{~N}=19$ & $\mathrm{~N}=6$ & $\mathrm{~N}=6$ & $\mathrm{~N}=3$ \\
\hline 1 & $100 \%$ & $83,3 \%$ & $18,2 \%$ & $15,7 \%$ & $16,6 \%$ & $16,6 \%$ & $0 \%$ \\
\hline 2 & & $100 \%$ & $72,2 \%$ & $21 \%$ & $50 \%$ & $16,6 \%$ & $0 \%$ \\
\hline 3 & & & $90,9 \%$ & $36,8 \%$ & $83 \%$ & $50 \%$ & $33,3 \%$ \\
\hline
\end{tabular}

Número total de pacientes: 59. 
La mayoría de los tratamientos realizados en nuestro servicio fueron ambulatorios, pudiendo en el mismo día desplazarse a su domicilio. Las necesidades anestésicas y analgésicas fueron mínimas. Igual que otros autores, la anestesia general sólo la empleamos en el tratamiento de niños (3).

En general no llegamos a utilizar ningún tipo de analgesia, si bien, otros autores, indican que con la utilización de pseudo-analgesia con petidina y midazolán se puede aumentar la potencia y el número de ondas, mejorando, la efectividad del tratamiento. A nuestro entender, el éxito de fragmentación no cambió sustancialmente al aumentar la potencia, y por otro lado podemos poner al paciente en riesgo de complicaciones, aumentando su morbilidad y el riesgo de "calle litiásica".

Igual que otros autores los kilovoltajes (dv) medios empleados, entre 15 y 28 con una media de 20,03 , y el tiempo por sesión que se sitúa en torno a 75 minutos son superponibles a los trabajos publicados para esta máquina $(3,5-7)$.

En los cálculos renales de hasta $1 \mathrm{~cm}$ tuvimos un porcentaje de éxito del $80.5 \%$ con única sesión, en dos sesiones fue del $92,2 \%$ y en tres sesiones $97.5 \%$. Para cálculos entre 1 y $2 \mathrm{~cm}$ el porcentaje de éxito disminuye al $48.2 \%$ en una única sesión, en dos sesiones fue del $75,4 \%$ y en tres sesiones del $87,3 \%$. Para cálculos entre 2 y $3 \mathrm{~cm}$ tratamos en única sesión el $29 \%$ de los pacientes, en dos sesiones el $52,3 \%$ y en tres sesiones el 66,5\%. A partir de $3 \mathrm{~cm}$ el índice de retratamientos aumenta considerablemente. Hasta cálculos de 2-3 cm el índice de éxito es muy bueno, a partir de cálculos de mayor tamaño el índice de éxito disminuye considerablemente. El estudio depende del tamaño del cálculo, sin olvidarnos como indican otros autores que la localización dentro de los cálices, la anatomía y fisiología de los propios cálices así como la posible naturaleza del cálculo influirán en el éxito del tratamiento (10).

Consideramos cálculo coraliforme, aquel que se localiza en la pelvis renal alcanzando uno, dos o varios brazos caliciales. En los cálculos de esta categoría colocamos "pig-tail" para evitar calle litiásica $(8,9)$. Efectuamos litotricia en 215 pacientes con cálculos coraliformes. Para nosotros el tamaño si influyó en el número de sesiones de litotricia. Si bien es cierto que tratamos cálculos de $3 \mathrm{~cm}$ en cinco sesiones y un cálculo de $10 \mathrm{~cm}$ en 6 sesiones, la media de sesiones por paciente fue de 7,25.

En general cálculos entre 2 y $3 \mathrm{~cm}$ hubo $100 \%$ de éxito en 5 sesiones, para cálculos entre 3 y $4 \mathrm{~cm} 72 \%$ fragmentaron en cinco sesiones, en cálculos entre 4 y $5 \mathrm{~cm}$ hubo una respuesta del $61 \%$ en cinco sesiones, entre 5 y $6 \mathrm{~cm} 60 \%$ de respuesta, entre 6 y $7 \mathrm{~cm} 51 \%$, entre 7 y $8 \mathrm{~cm} 37,5 \%$ de éxito.

Al aumentar el tamaño del cálculo fue necesario mayor número de sesiones. Según otros autores además del tamaño, el índice de éxito estaría relacionado con otros factores como la morfología del sistema calicial así como la dureza del cálculo (10). Esos factores no fueron considerados en nuestro estudio.

Realizamos una primera sesión y si respondian con fragmentación realizábamos sesiones sucesivas con un intervalo de tiempo de 2-3 semanas. Hay autores que indican realizar litotricia con generadores piezoeléctricos con intervalos de tiempo menores ya que no existe riesgo de complicaciones y el éxito de tratamiento es mayor, $(8,9)$. No tuvimos complicaciones mayores con respecto al tratamiento

TABLA IV. TAMAÑO CÁlCULO CORALIFORME.

\begin{tabular}{|c|c|c|c|c|c|c|c|}
\hline$N^{\circ}$ SESIONES & $\mathbf{2 0 - 2 9} \mathbf{~ m m}$ & $\mathbf{3 0 - 3 9} \mathbf{~ m m}$ & $\mathbf{4 0 - 4 9} \mathbf{~ m m}$ & $\mathbf{5 0 - 5 9} \mathbf{~ m m}$ & $\mathbf{6 0 - 6 9} \mathbf{~ m m}$ & $\mathbf{7 0 - 7 9} \mathbf{~ m m}$ & $\mathbf{8 0 - 8 9} \mathbf{~ m m}$ \\
\hline & $\mathrm{N}=4$ & $\mathrm{~N}=29$ & $\mathrm{~N}=59$ & $\mathrm{~N}=46$ & $\mathrm{~N}=31$ & $\mathrm{~N}=32$ & $\mathrm{~N}=12$ \\
\hline 1 & $25 \%$ & $13,8 \%$ & $13,5 \%$ & $6,5 \%$ & $12,9 \%$ & $12,5 \%$ & $0 \%$ \\
\hline 2 & $50 \%$ & $37,9 \%$ & $28,8 \%$ & $15,2 \%$ & $22,6 \%$ & $25 \%$ & $0 \%$ \\
\hline 3 & $75 \%$ & $44,8 \%$ & $50,8 \%$ & $19,5 \%$ & $25,8 \%$ & $31,2 \%$ & $8,3 \%$ \\
\hline & $75 \%$ & $55,2 \%$ & $59,3 \%$ & $41,3 \%$ & $35,5 \%$ & $37,5 \%$ & $8,3 \%$ \\
\hline & $100 \%$ & $72,4 \%$ & $61 \%$ & $60,8 \%$ & $51,6 \%$ & $37,5 \%$ & $8,3 \%$ \\
\hline
\end{tabular}

Número total de pacientes: 213. 
de este tipo de cálculos realizándose en ambulatorio, los pacientes recibieron profilaxia con antibiótico. Concluimos que la fragmentación de estos cálculos en monoterapia es difícil, quizás la utilización de nefrolitotomía percutánea conjuntamente con litotricia extracorpórea sea una alternativa más eficaz. Sin embargo, se podía intentar una primera sesión de litotricia para observar el comportamiento de fragmentación, ya que existen cálculos con un índice de dureza mínimo (cálculos de estruvita) y podríamos estar tratando pacientes con tratamientos de mayor morbilidad.

Tratamos 1.560 cálculos ureterales hasta un tamaño de $3 \mathrm{~cm}$, tuvimos una media de sesiones por paciente de 1,93. En tres sesiones fragmentamos $94,8 \%$ de todos los cálculos independientemente del tamaño. Al aumentar su tamaño se requirió de más sesiones de litotricia o de otro tipo de intervención. No aportamos la localización dentro del ureter, pero para algunos autores esto no influye en el éxito del tratamiento, siendo lo más importante el tamaño el tamaño del cálculo (11).

En cálculos vesicales aumentamos el número de ondas choque y la tensión por onda de choque hasta un máximo de 28 kv, el paciente se posicionó en decúbito prono, efectuamos litotricia de cálculos hasta un tamaño de $7 \mathrm{~cm}$, con una media de sesión de litotricia por paciente de 3,9. Al aumentar el tamaño del cálculo aumentó el número de sesiones.

En total tuvimos calle litiásica en 43 pacientes de todos los casos tratados, concluímos que para evitarlo sería importante la colocación de cateter ureteral en cálculos mayores de 2,5 cm, y la realización de la litotricia imponiendo tensiones de ondas bajas inicialmente para observar el comportamiento de fragmentación del cálculo, para posteriormente continuar con tensiones mayores o menores dependiendo del tal comportamiento. Para cálculos voluminosos localizados a nivel renal sería importante comenzar la fragmentación periféricamente para después intentar fragmentar el núcleo del cálculo.

\section{CONCLUSIÓN}

El litotritor MFL-5000 ha permitido el tratamiento de la litiasis urinaria en tod as las edades, con unos porcentajes de retratamiento y un número de tratamientos por cálculo similar a otros autores, sin necesidad de analgesia ni medidas anestésicas, salvo en aquellos pacientes de menor edad.

\section{COMENTARIO EDITORIAL}

Me han encomendado hacer una pequeña reseña sobre un trabajo efectuado con un Litotritor con el que hemos "cohabitado" durante 14 años y del que guardamos un agradable recuerdo, se trata del Litotritor MFL-5000, la tercera generación de Dornier y uno de los más completos de la serie.

Sin duda, es un trabajo donde las indicaciones del tratamiento tienen muy buen criterio y también el número de impactos por sesión y el kilo-voltaje utilizado.

Me llama la atención la falta de uso de analgésicos en gran parte de los pacientes sometidos al tratamiento, nosotros no lo hemos conseguido sin analgesia/sedación.

Respecto a los cálculos coraliformes necesitan un apartado aparte ya que su tratamiento es muy peculiar, no es lo mismo un cálculo coraliforme incompleto, un coraliforme completo ó un coraliforme completo y complejo y por otro lado, el tratamiento varía dependiendo de la composición del mismo, es decir, no es igual un cálculo de estruvita que un cálculo de oxalato cálcico.

El kilovoltaje utilizado es similar a otros autores, incluyendo nuestro grupo, aunque en los niños nosotros nunca superamos los $12 \mathrm{kw}$ y un máximo de 2.000 impactos por sesión.

Echo en falta la no hidratación renal, es decir, la no administración de diuréticos (furosemina) peroperatoria, la cual tiene dos objetivos en primer lugar la protección renal de los impactos y en segundo lugar el arrastre que ejerce el diurético sobre los fragmentos.

Tampoco puedo evitar "mi critica" sobre la "no indicación del ureteroscopio rígido, no sólo como instrumento complementario de la litotricia, sino como primera indicación en los cálculos del uréter distal".

Jorge Massarra.

Unidad de Urología.

Clínica La Luz. Madrid. 


\section{BIBLIOGRAFÍA y LECTURAS RECOMENDADAS ( ${ }^{*}$ lectura de interés $y^{* *}$ lectura fundamental)}

1. CHAUSSY, C.; BRENDEL, W.; SCHMIEDT, E.: "Extracorporeally induced destruction of kidney stones by shock waves". Lancet, 2: 1265, 1980.

2. SY, F.Y.; WONG, M.Y.C.; FOO, K.T.: "Current indication for open stone surgery in Singapore". Ann. Acad. Med. Singapore., 28: 241, 1999.

**3. MONTESINO, M.; SANTIAGO, A.; MILLÁN, J.A. y cols.: "Cinco años de experiencia en el tratamiento de la litiasis urinaria con el litotritor extracorpóreo MFL-5000". Anales Sistema Sanitario de Navarra. 21:2, 1998.

*4. SANTIAGO GONZÁLEZ DE GARIBAY, A.; MILLÁN SERRANO, J.A.; JIMÉNEZ CALVO, J. y cols.: "Tratamiento ambulatorio de la litiasis urinaria mediante litotricia extracorpórea (Dornier MFL-5000):estudio comparativo de su utilización a bajos y altos voltajes". Arch. Esp. Urol., 47: 151, 1994.

5. DENSTEDT, J.D.; SALES, J.L.; VALLELY, J.F. y cols.: "Experience with Dornier MFL-5000 extracorporeal shock wave lithotripsy". J. Urol., 147: 220, 1992.

6. DRACH, G.: "MFL 5000 Cooperative Study Group. Lithotripsy results utilizing a multifunctional urological unit”. J. Urol., 143: 377, 1990.

*7. CHAN, S.L.; STOTHERS, L.; ROWLEY, A. y cols.: "A prospective trial comparing the efficacy and complications of the modified HM-3 and MFL-5000 lithotriptors for solitary renal calculi". J. Urol., 153: 1794, 1995.

8. BRUNS, T.; STEIN, J.; TAUBER, R.: "Extracorporeal piezolectric shock wave lithotripsy as mono and multiple therapy of large renal calculi including staghorn sones in unanaesthetized patients under semi-ambulant conditions". British Journal of Urology, 75: 435, 1995.

*9. ACKERMANN, D.; CLAUS, R.; ZEHNTNER, C.H. y cols.: "Extracorporeal shock wave lithotripsy for large renal stones". Eur. Urol., 15: 5, 1988.

10. RASSWIELER, J.; GUMPINGER, R.; MILLER, K. y cols.: "Multimodal treatment (extracorporeal shock wave lithotripsy and endourology) of complicated reanl stone disease". Eur. Urol., 12: 294, 1986.

11. TAN, Y.M.; YIP, S.K.; CHONG, T.W. y cols.: "Clinical experience and results of ESWL treatment for 3093 urinary calculi with the Storz Modulith SL 20 lithotripter at the Singapore General Hospital". Scand. J. Urol. Nephrol., 36: 363, 2002. 